Article

\title{
Synthesis and Biological Evaluation of Carbocyclic Analogues of Pachastrissamine
}

\author{
Yongseok Kwon, Jayoung Song, Hoon Bae, Woo-Jung Kim, Joo-Youn Lee, Geun-Hee Han, \\ Sang Kook Lee and Sanghee Kim *
}

College of Pharmacy, Seoul National University, Seoul 151-742, Korea;

E-Mails: chemys@snu.ac.kr (Y.K.); poppy27@snu.ac.kr(J.S.); hoonbae1234@gmail.com (H.B.); lwjkim@snu.ac.kr (W.-J.K.); leejooyoun@snu.ac.kr(J.-Y.L.); thariel@snu.ac.kr (G.-H.H.); sklee61@snu.ac.kr (S.K.L.)

* Author to whom correspondence should be addressed; E-Mail: pennkim@snu.ac.kr; Tel.: +82-2-880-2487; Fax: +82-2-888-0649.

Academic Editors: Friedemann Honecker and Sergey A. Dyshlovoy

Received: 8 January 2015 / Accepted: 26 January 2015 / Published: 3 February 2015

\begin{abstract}
A series of carbocyclic analogues of naturally-occurring marine sphingolipid pachastrissamine were prepared and biologically evaluated. The analogues were efficiently synthesized via a tandem enyne/diene-ene metathesis reaction as a key step. We found that the analogue $\mathbf{4 b}$ exhibited comparable cytotoxicity and more potent inhibitory activity against sphingosine kinases, compared to pachastrissamine. Molecular modeling studies were conducted to provide more detailed insight into the binding mode of $\mathbf{4 b}$ in sphingosine kinase. In our docking model, pachastrissamine and $\mathbf{4 b}$ were able to effectively bind to the binding pocket of sphingosine kinase 1 as co-crystalized sphingosine. However, $\mathbf{4 b}$ showed a hydrophobic interaction with Phe192, which suggests that it contributes to its increased inhibitory activity against sphingosine kinase 1.
\end{abstract}

Keywords: pachastrissamine; jaspine B; carbocyclic analogue; cytotoxicity; sphingosine kinase inhibitor; molecular modeling

\section{Introduction}

Pachastrissamine (jaspine B, 1; Figure 1) is a natural anhydrous derivative of phytosphingosine 2, possessing a tetrahydrofuran core with three contiguous stereogenic centers. It was first isolated by 
Higa et al. from marine sponge Pachastrissa sp. in 2002 [1] and subsequently isolated from the Vanuatuan marine sponge Jaspis sp. by Debitus et al. in 2003 [2]. This marine natural product showed significant cytotoxicity against various cancer cell lines, such as P388, MEL28, A549, HT29 and $\mathrm{HeLa}[1,3]$. It was found to have an inhibitory activity against sphingomyelin synthase, which, in turn, triggers apoptosis in tumor cells via a caspase-dependent pathway [4]. It was also reported that pachastrissamine and its stereoisomers inhibit sphingosine kinases (SphKs) and atypical protein kinase C [5]. Because of its intriguing biological activity, it has been an interesting target for synthetic chemists, and various synthetic routes to pachastrissamine have been reported [6-16]. However, the structure-activity relationship (SAR) of pachastrissamine remains relatively unreported. Génisson et al. described analogues with a modified aliphatic chain, which exhibited cytotoxicity comparable to or lower than pachastrissamine on two distinct cancer cell lines (B16 and A375 melanoma cell lines) $[17,18]$. Delgado et al. reported the stereoisomers of pachastrissamine as being 10- to 20-times less potent than the natural one [19]. Recently, Liu et al. reported pachastrissamine analogues containing a 1,2,3-triazole ring in the alkyl chain [20]. One analogue showed better increased cytotoxicity than the natural compound. These results suggest that the configuration and aliphatic chain of pachastrissamine would be essential to retain biological activity. The contribution of the tetrahydrofuran ring to its biological profile has been explored. The aza-analogues of pachastrissamine 3a were reported by Génisson et al. [21], and the sulfur and selenium analogues $\mathbf{3 b}-\mathbf{c}$ were previously reported by our group [22]. They exhibited comparable potency to natural pachastrissamine, indicating that the ring oxygen atom of pachastrissamine could be replaced with bioisosteres.

In this regard, we designed carbocyclic analogues, replacing the ethereal oxygen $(-\mathrm{O}-)$ with a methylene group $\left(-\mathrm{CH}_{2}-\right)$ as a divalent bioisostere [23]. The advantages of carbocyclic analogues of natural product have already been demonstrated by several successful examples, such as carbasugars and carbocyclic nucleosides [24,25]. As seen in these successful precedents, we expected that the carbocyclic analogue would show enhanced chemical and metabolic stability compared to the parent natural product. In addition, from this study, we would be able to decipher the SAR involved in the role of ethereal oxygen, which is valuable information in the development of new anti-cancer therapeutic agents. Herein, we report the synthesis and biological evaluation of a series of carbocyclic pachastrissamine analogues 4 (Figure 1) in which the alkyl-chain lengths have been varied.
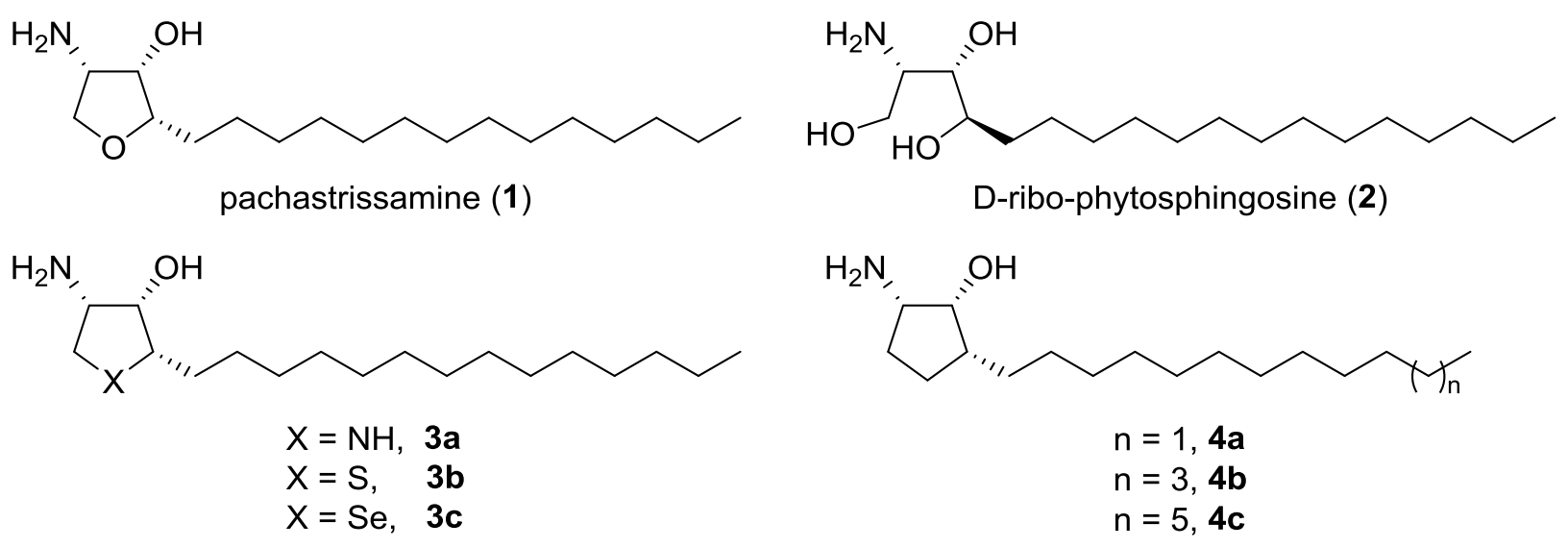

Figure 1. Chemical structures of Compounds 1-4. 


\section{Results and Discussion}

\subsection{Chemistry}

\subsubsection{Retrosynthetic Analysis}

Our retrosynthetic plan for the synthesis of $\mathbf{4}$ is shown in Scheme 1. The designed analogues $\mathbf{4}$ would be accessed from diene $\mathbf{5}$ using catalytic hydrogenation and hydrolysis. We expected catalytic hydrogenation of $\mathbf{5}$ would occur from the convex face of the bicyclic system to afford the desired stereochemistry [6]. The requisite diene 5 would be derived from the ring-closing metathesis (RCM) of enyne 6 and subsequent cross-metathesis (CM) between the resulting diene and the appropriate olefin in a tandem fashion [26-29]. Substrate 6 would be derived from a known amide 7, which, in turn, can be easily prepared from commercially available $(S)$-allylglycine.

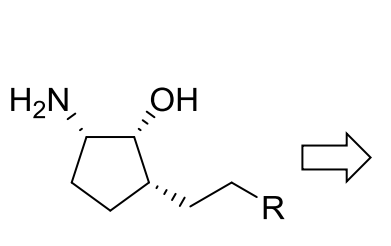

4<smiles>[R]C=CC1=CC[C@@H]2NC(=O)O[C@H]12</smiles>

5
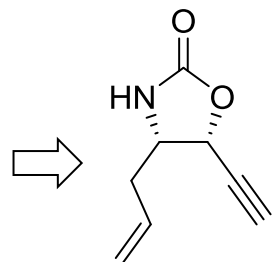

6

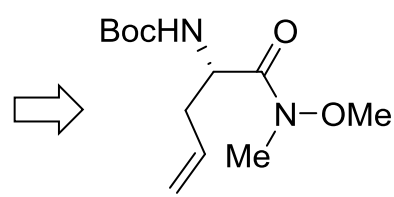

7

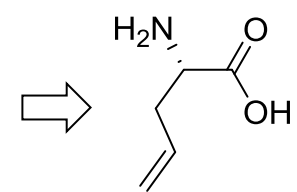

(S)-allylglycine

Scheme 1. Retrosynthetic plan.

\subsubsection{Synthesis}

Our synthesis, as shown in Scheme 2, commenced with the preparation of a known Weinreb amide 7 from commercially available $(S)$-allylglycine in two steps using a previously reported procedure [30]. Treatment of amide 7 with lithium acetylide in THF yielded ynone 8 in a $71 \%$ yield. The stereoselective reduction of ynone $\mathbf{8}$ was explored with various hydride reagents. After several trials, it was found that the treatment of $\mathbf{8}$ with sodium borohydride and cerium chloride at low temperature gave an inseparable diastereomeric mixture of alcohol $\mathbf{9}$ with the best selectivity (diastereomeric ratio $=6: 1$ ) in $87 \%$ yield. The obtained alcohol 9 was treated with lithium hexamethyldisilazide in hot toluene to provide oxazolidinone $\mathbf{1 0}$ in a high yield (90\%). At this stage, both diastereomers (10a and $\mathbf{1 0 b})$ were separated via column chromatography, and the relative configuration of the diastereomers was assigned by a 2D-NOESY experiment (Figures S7 and S10 in the Supplementary Materials). The triisopropylsilyl group of major diastereomer 10a was easily removed with tetrabutylammonium fluoride to give the terminal acetylene 6 in a $95 \%$ yield. 

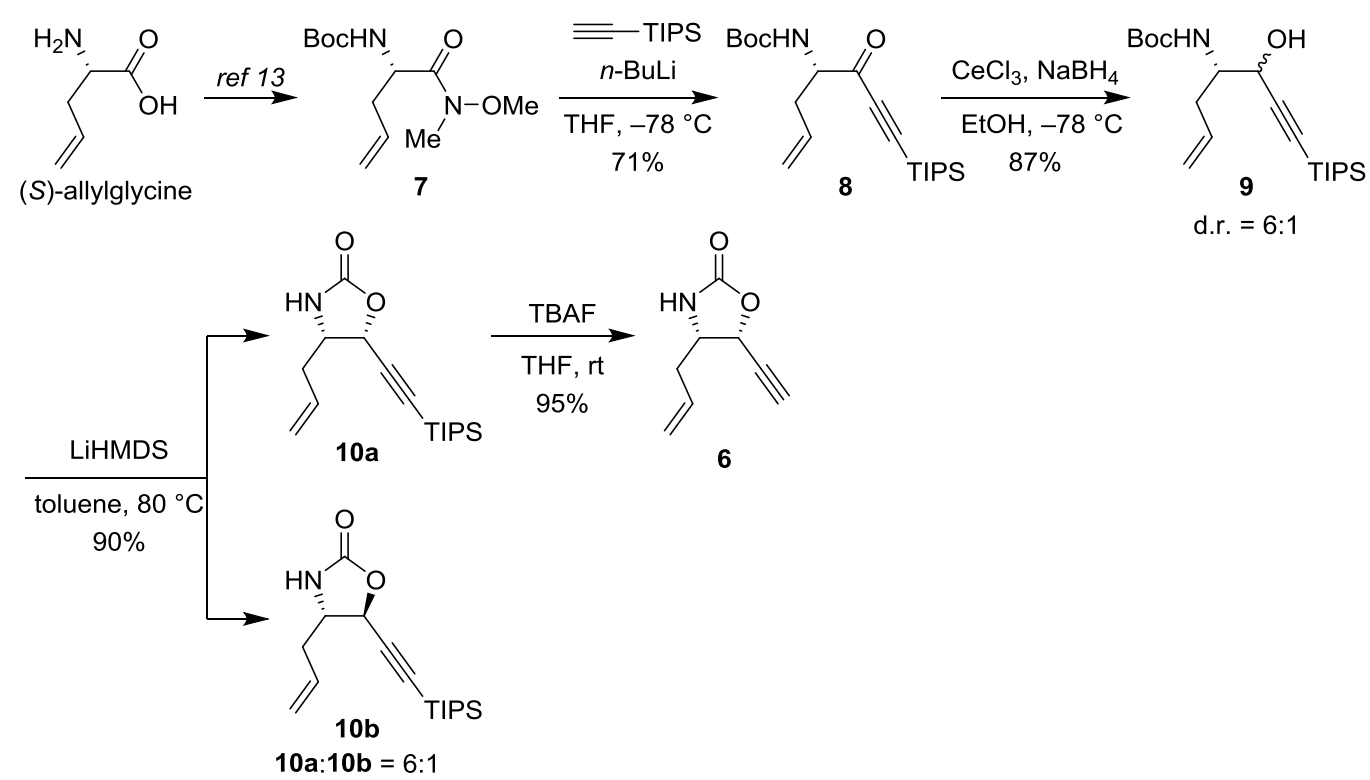

Scheme 2. Synthesis of enyne 6. TIPS: triisopropylsilyl; LiHMDS: lithium hexamethyldisilazide; TBAF: tetrabutylammonium fluoride.

With the terminal enyne 6 in hand, the tandem enyne/diene-ene metathesis reaction was carried out (Scheme 3). The reaction of 6 with the appropriate alkenes in the presence of second-generation Grubbs catalyst ( $3 \mathrm{~mol} \%$ ) afforded the desired diene 5a-c with exclusive $(E)$-geometry. The catalytic hydrogenation of diene $\mathbf{5 a - c}$ was accomplished to give cyclopentane 11a-c as the only stereoisomers detected by ${ }^{1} \mathrm{H}$ NMR. Finally, hydrolysis of the oxazolidinone with $\mathrm{KOH}$ in refluxing EtOH afforded the desired carbocyclic analogues, $\mathbf{4 a - c}$.

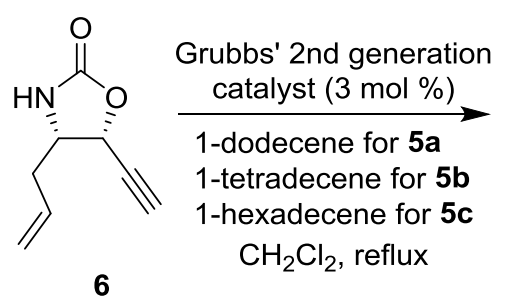

6

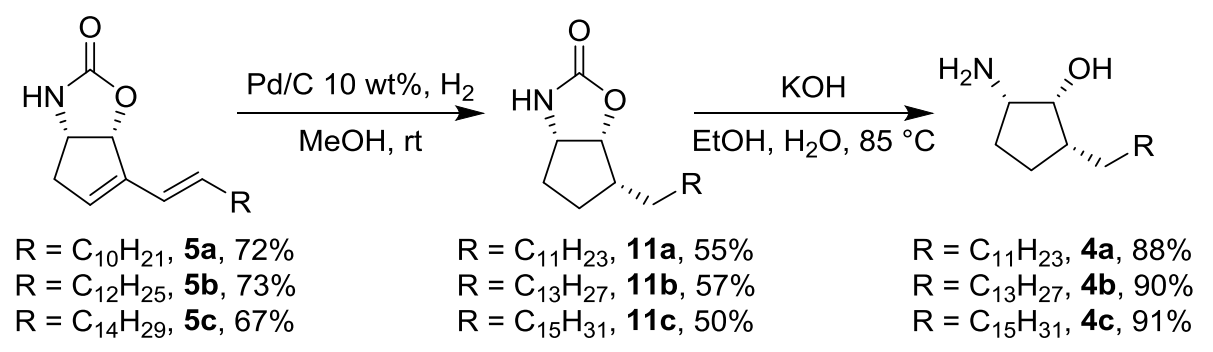

Scheme 3. Synthesis of carbocyclic analogues, $4 \mathbf{a}-\mathbf{c}$.

\subsection{Biological Evaluation}

\subsubsection{Cell Viability}

The cytotoxic activity of analogues $\mathbf{4 a - c}$ was examined in various cancer cell lines (Table 1) by the sulforhodamine B (SRB) assay [31]. For a direct comparison, natural pachastrissamine (1) was employed as a positive control. It was found that the cytotoxic activities of carbocyclic analogues 4 were influenced by the length of the alkyl chain. The analogue, $\mathbf{4 b}$, which has the same chain length as pachastrissamine, exhibited a comparable potency to the parent natural product, $\mathbf{1}$, against various cell lines. However, both the shorter and longer chain analogues, 4a and $\mathbf{4 c}$, were less effective than pachastrissamine. 
Table 1. Cytotoxic activity of pachastrissamine (1) and its carbocyclic analogues, $\mathbf{4 a - c .}$

\begin{tabular}{cccccc}
\hline \multirow{2}{*}{ Compound } & \multicolumn{5}{c}{ IC $_{\mathbf{5 0}}(\boldsymbol{\mu M})^{\mathbf{a}}$} \\
\cline { 2 - 6 } & $\mathbf{H C T - 1 1 6}^{\mathbf{b}}$ & $\mathbf{S N U - 6 3 8}^{\mathbf{c}}$ & MDA-MB-231 $^{\mathbf{d}}$ & PC-3 $^{\mathbf{e}}$ & Caki-1 $^{\mathbf{f}}$ \\
\hline $\mathbf{1}$ & 1.0 & 1.2 & 0.7 & 0.7 & 1.7 \\
$\mathbf{4 a}$ & 9.7 & 12.8 & 6.1 & 7.6 & 12.1 \\
$\mathbf{4 b}$ & 1.0 & 1.7 & 1.8 & 1.0 & 3.2 \\
$\mathbf{4 c}$ & 3.2 & 4.4 & 4.1 & 3.1 & 6.6 \\
\hline
\end{tabular}

a $50 \%$ inhibition concentration; ${ }^{b}$ HCT-116, human colon cancer cell line; ${ }^{c}$ SNU-638, human stomach cancer cell line; ${ }^{\mathrm{d}} \mathrm{MDA}-\mathrm{MB}-231$, human breast cancer cell line; ${ }^{\mathrm{e}} \mathrm{PC}-3$, human prostate cancer cell line;

${ }_{\mathrm{f}}^{\mathrm{f}}$ Caki-1, human renal cancer cell line.

\subsubsection{Inhibitory Activity against Sphingosine Kinases}

Because pachastrissamine was reported as an inhibitor of sphingosine kinases (SphKs), the inhibitory activity of analogues 4 against SphKs were examined using a sphingosine kinase inhibition assay (Table 2). There are two isoforms of mammalian SphKs (SphK1 and SphK2) catalyzing the phosphorylation of sphingosine to sphingosine 1-phosphate (S1P). S1P regulates diverse cellular processes, including cell growth, survival and differentiation [32]. In our experiments, $\mathrm{N}, \mathrm{N}$-dimethylsphingosine (DMS), as well as pachastrissamine (1) were used as a positive control [33]. It was revealed that the inhibitory activity of $\mathbf{4}$ against SphKs was influenced by the length of the attached chain. The analogue $\mathbf{4 b}$ showed a similar inhibitory activity to DMS, which, in turn, was more effective than 1. However, the other analogues, $\mathbf{4 a}$ and $\mathbf{4 c}$, were found to be less effective at the inhibition of SphKs. These results suggest that the ring oxygen atom of pachastrissamine is dispensable, but the appropriate chain length is apparently required to retain its biological properties.

Table 2. Inhibitory activity of pachastrissamine (1) and its carbocyclic analogues, $\mathbf{4 a - c}$, against sphingosine kinases.

\begin{tabular}{ccc}
\hline \multirow{2}{*}{ Compound } & \multicolumn{2}{c}{$\mathbf{I C}_{\mathbf{5 0}}(\boldsymbol{\mu M})^{\text {a }}$} \\
\cline { 2 - 3 } & $\mathbf{S p h K 1}$ & SphK2 \\
\hline $\mathbf{1}$ & 12.0 & 41.8 \\
$\mathbf{4 a}$ & 58.2 & $>100$ \\
$\mathbf{4 b}$ & 7.5 & 20.1 \\
$\mathbf{4 c}$ & 41.3 & $>100$ \\
DMS $^{\mathrm{b}}$ & 6.6 & 19.9 \\
\hline 50\% inhibition concentration; ${ }^{\mathrm{b}} N, N$-dimethylsphingosine.
\end{tabular}

\subsection{Molecular Modeling}

To understand the molecular interaction between $\mathbf{4 b}$ and the sphingosine kinase, a molecular modeling study was performed using the crystal structure of human SphK1 in complex with sphingosine (PDB Code 3VZB) [34]. As shown in Figure 2, pachastrissamine (1) and its carbocyclic analogue $\mathbf{4 b}$ are able to effectively bind to the binding pocket of SphK1 in virtually the same pose. They occupy nearly the same position as sphingosine. In our docking model, the alkyl chain of $\mathbf{4 b}$ is buried in the hydrophobic J-shaped tunnel constituted of hydrophobic residues Leu268, Ala274, 
His311, Phe173, Phe303, Val177 and Phe192. The hydroxyl group forms a hydrogen bond with Asp178 and a water-mediated hydrogen bond with Ser168, in the same manner as the 3-hydroxyl group of sphingosine. The amino group makes a hydrogen bond interaction with Ser168. In the hydrophilic recognition site, pachastrissamine (1) and its analogue $\mathbf{4 b}$ formed the same set of hydrogen bonds. However, carbocyclic analogue $\mathbf{4 b}$ exhibits an additional interaction with the hydrophobic residue, Phe192 (Figure 2B). This pi-alkyl interaction seems to contribute to the increased activity of 4b toward SphK1 compared to 1 [35].
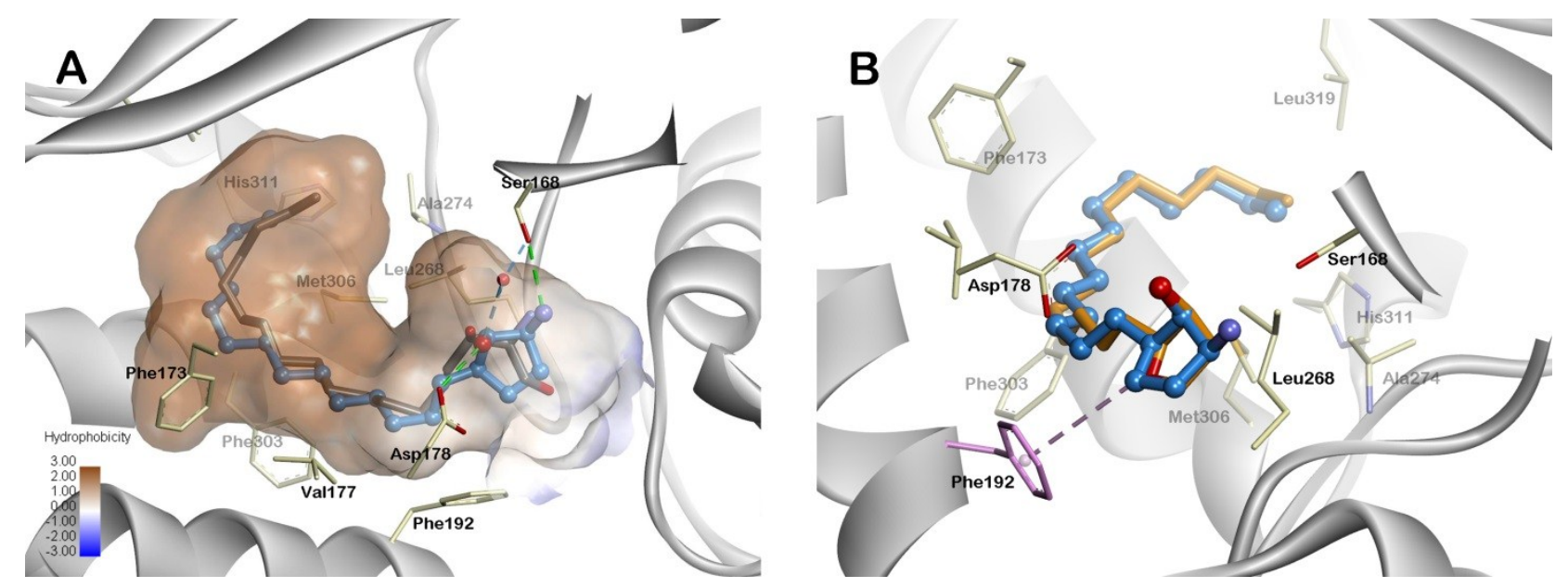

Figure 2. Docking model of the carbocyclic analogue $\mathbf{4 b}$ and pachastrissamine (1). (A) The overlay of the proposed binding model of carbocyclic analogue $\mathbf{4 b}$ (blue) and $\mathrm{X}$-ray complex sphingosine (gray). A water molecule is shown as a red sphere. The direct H-bonds and water-mediated H-bonds are shown as green and blue dashed lines, respectively. The ligand binding surface of the binding pocket was colored according to hydrophobicity (brown: hydrophobic, blue: hydrophilic); (B) The overlay of the proposed binding model of $\mathbf{4 b}$ (blue) and $\mathbf{1}$ (orange). Hydrophobic interaction with Phe192 is shown as a pink dashed line.

\section{Experimental Section}

\subsection{Chemistry}

\subsubsection{General}

All chemicals were reagent grade and used as purchased. All reactions were performed under an inert atmosphere of dry nitrogen using distilled dry solvents. Reactions were monitored through thin layer chromatography (TLC) analysis using silica gel 60 F-254 thin layer plates. Compounds were visualized on the TLC plates under UV light and by spraying with either $\mathrm{KMnO}_{4}$ or anisaldehyde staining solutions. Flash column chromatography was conducted on silica gel 60 (230-400 mesh). Melting points were measured using a Buchi B-540 melting point apparatus without correction. ${ }^{1} \mathrm{H}$ NMR (400, 500 or $\left.600 \mathrm{MHz}\right)$ and ${ }^{13} \mathrm{C}$ NMR $(100,125$ or $150 \mathrm{MHz}$ ) spectra were recorded in $\delta$ units relative to the deuterated solvent. The IR spectra were measured on a Fourier transform infrared spectrometer. High-resolution mass spectra (HRMS) were recorded using FAB. 


\subsection{2. tert-Butyl (S)-(5-oxo-7-(triisopropylsilyl)hept-1-en-6-yn-4-yl)carbamate (8)}

To a solution of (triisopropylsilyl)acetylene $(17.4 \mathrm{~mL}, 77.4 \mathrm{mmol})$ in THF $(80 \mathrm{~mL})$ was added $n$-BuLi (48.4 mL, $77.4 \mathrm{mmol}, 1.6 \mathrm{M}$ solution in hexane) at $-78{ }^{\circ} \mathrm{C}$ under nitrogen. After the reaction mixture was stirred for $30 \mathrm{~min}$ at $-78^{\circ} \mathrm{C}$, known amide $7(10 \mathrm{~g}, 38.7 \mathrm{mmol})$ in dry THF $(20 \mathrm{~mL})$ was added at $0{ }^{\circ} \mathrm{C}$. The reaction mixture was stirred for $1.5 \mathrm{~h}$ at $0{ }^{\circ} \mathrm{C}$. The reaction was quenched with a saturated $\mathrm{NH}_{4} \mathrm{Cl}$ aqueous solution and extracted with EtOAc. The organic layer was washed twice with brine, dried over $\mathrm{MgSO}_{4}$, filtered and concentrated. The crude product was purified using column chromatography on silica gel (hexane/EtOAc, 20:1), to afford the desired ynone 8 (10.4 g, 71\%) as a yellow oil. $[\alpha]_{\mathrm{D}}^{20}+8.20\left(\mathrm{c} 1.0, \mathrm{CHCl}_{3}\right) ;{ }^{1} \mathrm{H}$ NMR $\left(400 \mathrm{MHz}, \mathrm{CDCl}_{3}\right) \delta 5.70-5.61(\mathrm{~m}, 1 \mathrm{H}), 5.13(\mathrm{~d}$, $J=17.2 \mathrm{~Hz}, 1 \mathrm{H}), 5.12(\mathrm{~d}, J=9.2 \mathrm{~Hz}, 1 \mathrm{H}), 5.10$ (brs, $1 \mathrm{H}), 4.48$ (d, $J=5.7 \mathrm{~Hz}, 1 \mathrm{H}), 2.70-2.59(\mathrm{~m}, 2 \mathrm{H})$, $1.42(\mathrm{~s}, 9 \mathrm{H}), 1.17-1.01(\mathrm{~m}, 21 \mathrm{H}) ;{ }^{13} \mathrm{C} \mathrm{NMR}\left(100 \mathrm{MHz}, \mathrm{CDCl}_{3}\right) \delta 185.4,155.1,131.7,119.5,102.2$, 100.1, 80.0, 60.8, 36.0, 28.3 (3C), 18.5 (6C), 11.0 (3C); IR ( $\left.\mathrm{CHCl}_{3}\right) v_{\max } 3358,2947,2869,2150$, 1719, 1682, 1495, 1368, $1171\left(\mathrm{~cm}^{-1}\right)$; HRMS (FAB) calcd. for $\mathrm{C}_{21} \mathrm{H}_{38} \mathrm{NO}_{3} \mathrm{Si}\left([\mathrm{M}+\mathrm{H}]^{+}\right), 380.2621$; found, 380.2614 .

\subsection{3. tert-Butyl ((4S)-5-hydroxy-7-(triisopropylsilyl)hept-1-en-6-yn-4-yl)carbamate (9)}

To a solution of ynone $8(10 \mathrm{~g}, 26.3 \mathrm{mmol})$ in $\mathrm{EtOH}(100 \mathrm{~mL})$ was added anhydrous $\mathrm{CeCl}_{3}(13 \mathrm{~g}$, $52.6 \mathrm{mmol}$ ) at room temperature under nitrogen. After the reaction mixture was stirred for $15 \mathrm{~min}$, $\mathrm{NaBH}_{4}(2.0 \mathrm{~g}, 52.6 \mathrm{mmol})$ was added at $-78{ }^{\circ} \mathrm{C}$. The reaction mixture was stirred for $3 \mathrm{~h}$ at $-78{ }^{\circ} \mathrm{C}$. The reaction was quenched with a saturated $\mathrm{NH}_{4} \mathrm{Cl}$ aqueous solution and extracted with EtOAc. The organic layer was washed twice with brine, dried over $\mathrm{MgSO}_{4}$, filtered and concentrated. The crude product was purified using column chromatography on silica gel (hexane/EtOAc, 5:1), to afford the desired alcohol $9(8.7 \mathrm{~g}, 87 \%)$ as a diastereomeric mixture $(6: 1)$ as a colorless oil. $[\alpha]_{\mathrm{D}}^{20}-18.3\left(\mathrm{c} 1.0, \mathrm{CHCl}_{3}\right) ;{ }^{1} \mathrm{H}$ NMR $\left(400 \mathrm{MHz}, \mathrm{CDCl}_{3}\right) \delta 5.82-5.72(\mathrm{~m}, 1 \mathrm{H}), 5.08(\mathrm{~d}, J=15.2 \mathrm{~Hz}$, $1 \mathrm{H}), 5.06(\mathrm{~d}, J=8.9 \mathrm{~Hz}, 1 \mathrm{H}), 4.74-4.72(\mathrm{~m}, 0.85 \mathrm{H}), 4.45-4.44(\mathrm{~m}, 1 \mathrm{H}), 3.82$ (brs, $0.85 \mathrm{H}), 3.72$ (brs, $0.15 \mathrm{H}), 3.38$ (brs, 0.85H), 2.56-2.46 (m, 0.15H), 2.42-2.22 (m, 1.85H), $1.40(\mathrm{~s}, 9 \mathrm{H}), 1.04(\mathrm{~s}, 21 \mathrm{H})$; ${ }^{13} \mathrm{C} \mathrm{NMR}\left(100 \mathrm{MHz}, \mathrm{CDCl}_{3}\right) \delta 156.5,134.0,118.0,105.2,87.6,79.9,66.1,55.1,35.7,28.2(3 \mathrm{C})$, 18.5 (6C), 11.1 (3C); IR $\left(\mathrm{CHCl}_{3}\right) v_{\max } 3428,2945,2868,2170,1696,1504,1170\left(\mathrm{~cm}^{-1}\right)$; HRMS (FAB) calcd. for $\mathrm{C}_{21} \mathrm{H}_{40} \mathrm{NO}_{3} \mathrm{Si}\left([\mathrm{M}+\mathrm{H}]^{+}\right), 382.2777$; found, 382.2770.

\subsection{4. (4S)-4-Allyl-5-((triisopropylsilyl)ethynyl)oxazolidin-2-one (10)}

To a solution of alcohol $9(8 \mathrm{~g}, 21 \mathrm{mmol})$ in toluene $(100 \mathrm{~mL})$ was added lithium hexamethyldisilazide $(46.2 \mathrm{~mL}, 46.2 \mathrm{mmol}, 1 \mathrm{M}$ solution in THF) at room temperature under nitrogen. After stirring for $1.5 \mathrm{~h}$ at $80{ }^{\circ} \mathrm{C}$, the reaction mixture was cooled to $0{ }^{\circ} \mathrm{C}$. The reaction was quenched with a saturated $\mathrm{NH}_{4} \mathrm{Cl}$ aqueous solution and extracted with EtOAc. The organic layer was washed twice with brine, dried over $\mathrm{MgSO}_{4}$, filtered and concentrated. The crude product was purified using column chromatography on silica gel (hexane/EtOAc, 1.5:1), to afford the desired oxazolidinone 10a $(5.0 \mathrm{~g}, 77 \%)$ and $\mathbf{1 0 b}(850 \mathrm{mg}, 13 \%)$.

(4S,5R)-4-Allyl-5-((triisopropylsilyl)ethynyl)oxazolidin-2-one (10a): a white solid; m.p. 91.2-93.3 ${ }^{\circ} \mathrm{C}$; $[\alpha]_{\mathrm{D}}^{20}-4.12\left(\mathrm{c} 0.35, \mathrm{CHCl}_{3}\right) ;{ }^{1} \mathrm{H} \mathrm{NMR}\left(400 \mathrm{MHz}, \mathrm{CDCl}_{3}\right) \delta 5.80-5.72(\mathrm{~m}, 1 \mathrm{H}), 5.33-5.31(\mathrm{~d}$, 
$J=7.8 \mathrm{~Hz}, 1 \mathrm{H}), 5.20(\mathrm{~d}, J=10.4 \mathrm{~Hz}, 1 \mathrm{H}), 5.19$ (d, $J=16.8 \mathrm{~Hz}, 1 \mathrm{H}), 5.17$ (brs, 1H), 3.88 (td, $J=4.1 \mathrm{~Hz}, 8.6 \mathrm{~Hz}, 1 \mathrm{H}), 2.59-2.53(\mathrm{~m}, 1 \mathrm{H}), 2.44-2.36(\mathrm{~m}, 1 \mathrm{H}), 1.07(\mathrm{~s}, 21 \mathrm{H}) ;{ }^{13} \mathrm{C} \mathrm{NMR}(100 \mathrm{MHz}$, $\left.\mathrm{CDCl}_{3}\right) \delta 159.6,132.7,119.8,98.5,93.5,70.1,54.1,36.8,18.5$ (6C), $11.0(3 \mathrm{C})$; IR $\left(\mathrm{CHCl}_{3}\right) v_{\max } 3276$, 2946, 2868, 1759, 1464, 1334, 1048, 758, $676\left(\mathrm{~cm}^{-1}\right)$; HRMS (FAB) calcd. for $\mathrm{C}_{17} \mathrm{H}_{30 \mathrm{NO}_{2} \mathrm{Si}}$ $\left([\mathrm{M}+\mathrm{H}]^{+}\right), 308.2046$; found, 308.2039.

(4S,5S)-4-Allyl-5-((triisopropylsilyl)ethynyl)oxazolidin-2-one (10b): a yellow oil; $[\alpha]_{\mathrm{D}}^{20}-36.2$ (c 1.4 , $\left.\mathrm{CHCl}_{3}\right) ;{ }^{1} \mathrm{H} \mathrm{NMR}\left(600 \mathrm{MHz}, \mathrm{CDCl}_{3}\right) \delta 6.12(\mathrm{~d}, J=9.2 \mathrm{~Hz}, 1 \mathrm{H}), 5.77-5.70(\mathrm{~m}, 1 \mathrm{H}), 5.19(\mathrm{~d}, J=11.5 \mathrm{~Hz}$, $1 \mathrm{H}), 5.18(\mathrm{~d}, J=16.1 \mathrm{~Hz}, 1 \mathrm{H}), 4.80(\mathrm{~d}, J=6.4 \mathrm{~Hz}, 1 \mathrm{H}), 3.87(\mathrm{q}, J=6.4 \mathrm{~Hz}, 1 \mathrm{H}), 2.40$ (q, $J=6.7 \mathrm{~Hz}$, $1 \mathrm{H}), 2.33$ (q, $J=7.1 \mathrm{~Hz}, 1 \mathrm{H}), 1.05(\mathrm{~s}, 21 \mathrm{H}) ;{ }^{13} \mathrm{C} \mathrm{NMR}\left(150 \mathrm{MHz}, \mathrm{CDCl}_{3}\right) \delta 158.2,131.5,119.8$, 101.7, 90.8, 70.9, 59.2, 38.7, 18.5 (6C), 11.0 (3C); IR $\left(\mathrm{CHCl}_{3}\right) v_{\max } 3282,2946,2869,1760,1464$, 1323, 1071, 992, 883, $675\left(\mathrm{~cm}^{-1}\right)$; HRMS (FAB) calcd. for $\mathrm{C}_{17} \mathrm{H}_{30} \mathrm{NO}_{2} \mathrm{Si}\left([\mathrm{M}+\mathrm{H}]^{+}\right), 308.2046$; found, 308.2042.

\subsection{5. (4S,5R)-4-Allyl-5-ethynyloxazolidin-2-one (6)}

To a solution of alcohol 10a $(4.0 \mathrm{~g}, 13.0 \mathrm{mmol})$ in THF $(60 \mathrm{~mL})$ was added tetrabutylammonium fluoride (15.6 mL, $15.6 \mathrm{mmol}, 1 \mathrm{M}$ solution in THF) at room temperature under nitrogen. The reaction mixture was stirred for $1 \mathrm{~h}$ at room temperature. The reaction was quenched with a saturated $\mathrm{NH}_{4} \mathrm{Cl}$ aqueous solution and extracted with EtOAc. The organic layer was washed twice with brine, dried over $\mathrm{MgSO}_{4}$, filtered and concentrated. The crude product was purified using column chromatography on silica gel (hexane/EtOAc, 1:1), to afford the desired terminal acetylene $6(1.87 \mathrm{~g}, 95 \%)$ as a white solid. m.p. $42.0-44.0{ }^{\circ} \mathrm{C} ;[\alpha]_{\mathrm{D}}^{20}-6.32\left(\mathrm{c} 0.54, \mathrm{CHCl}_{3}\right) ;{ }^{1} \mathrm{H}$ NMR $\left(400 \mathrm{MHz}, \mathrm{CDCl}_{3}\right) \delta 5.81-5.70(\mathrm{~m}, 1 \mathrm{H})$, $5.28(\mathrm{dd}, J=2.2 \mathrm{~Hz}, 7.8 \mathrm{~Hz}, 2 \mathrm{H}), 5.22(\mathrm{~d}, J=0.7 \mathrm{~Hz}, 1 \mathrm{H}), 5.19-5.18(\mathrm{~m}, 1 \mathrm{H}), 3.91(\mathrm{td}, J=4.1 \mathrm{~Hz}$, $8.6 \mathrm{~Hz}, 1 \mathrm{H}) 2.72(\mathrm{~d}, J=2.1 \mathrm{~Hz}, 1 \mathrm{H}), 2.58-2.52(\mathrm{~m}, 1 \mathrm{H}), 2.44-2.36(\mathrm{~m}, 1 \mathrm{H}) ;{ }^{13} \mathrm{C} \mathrm{NMR}(150 \mathrm{MHz}$, $\left.\mathrm{CDCl}_{3}\right) \delta 157.2,132.4,120.0,79.0,75.8,69.3,53.9,36.5 ; \mathrm{IR}\left(\mathrm{CHCl}_{3}\right) v_{\max } 3292,2128,1755,1644$, 1338, 1243, $1041\left(\mathrm{~cm}^{-1}\right)$; HRMS (FAB) calcd. for $\mathrm{C}_{8} \mathrm{H}_{10} \mathrm{NO}_{2}\left([\mathrm{M}+\mathrm{H}]^{+}\right), 152.0712$; found, 152.0714 .

\subsubsection{General Procedure for the Preparation of 5}

To a solution of $6(300 \mathrm{mg}, 2.0 \mathrm{mmol})$ with the appropriate alkene $(2 \mathrm{~mL})$ in $\mathrm{CH}_{2} \mathrm{Cl}_{2}(20 \mathrm{~mL})$ was added Grubbs' second-generation catalyst $(50 \mathrm{mg}, 0.059 \mathrm{mmol})$ at room temperature. The resulting mixture was refluxed for $20 \mathrm{~h}$. After the mixture was cooled to room temperature, the solvent was removed under reduced pressure. The crude product was purified by column chromatography on silica gel (hexane/EtOAc, 1:3) to give diene 5.

\subsection{7. (3aS,6aR)-6-((E)-Dodec-1-en-1-yl)-3,3a,4,6a-tetrahydro-2H-cyclopenta[d]oxazol-2-one (5a)}

A white solid (420 mg, 72\%); m.p. 83.6-84.5 ${ }^{\circ} \mathrm{C}$; $[\alpha]_{\mathrm{D}}^{20}+23.7\left(\mathrm{c} 1.1, \mathrm{CHCl}_{3}\right) ;{ }^{1} \mathrm{H}$ NMR $(500 \mathrm{MHz}$, $\left.\mathrm{CDCl}_{3}\right) \delta 6.12(\mathrm{~d}, J=16.0 \mathrm{~Hz}, 1 \mathrm{H}), 5.94(\mathrm{td}, J=7.4 \mathrm{~Hz}, 15.2 \mathrm{~Hz}, 1 \mathrm{H}), 5.68(\mathrm{~s}, 1 \mathrm{H}), 5.63$ (brs, $\left.1 \mathrm{H}\right)$, $5.59(\mathrm{~d}, J=7.8 \mathrm{~Hz}, 1 \mathrm{H}), 4.44(\mathrm{t}, J=7.2 \mathrm{~Hz}, 1 \mathrm{H}), 2.71(\mathrm{dd}, J=6.1 \mathrm{~Hz}, 18.1 \mathrm{~Hz}, 1 \mathrm{H}), 2.47(\mathrm{~d}$, $J=18.2 \mathrm{~Hz}, 1 \mathrm{H}), 2.15-2.02(\mathrm{~m}, 2 \mathrm{H}), 1.40-1.35(\mathrm{~m}, 2 \mathrm{H}), 1.24(\mathrm{~s}, 14 \mathrm{H}), 0.86(\mathrm{t}, J=6.9 \mathrm{~Hz}, 3 \mathrm{H})$;

${ }^{13} \mathrm{C} \mathrm{NMR}\left(125 \mathrm{MHz}, \mathrm{CDCl}_{3}\right) \delta$ 158.9, 139.8, 135.0, 128.8, 123.0, 84.9, 53.7, 40.2, 33.1, 31.9, 29.62, 
29.58, 29.48, 29.33, 29.25, 29.0, 22.7, 14.1; IR $\left(\mathrm{CHCl}_{3}\right) v_{\max } 3270,2922,2854,1739,1716,1385$, 1238, 1018, $756\left(\mathrm{~cm}^{-1}\right)$; HRMS (FAB) calcd. for $\mathrm{C}_{18} \mathrm{H}_{30} \mathrm{NO}_{2}\left([\mathrm{M}+\mathrm{H}]^{+}\right), 292.2277$; found, 292.2279.

3.1.8. (3aS,6aR)-6-((E)-Tetradec-1-en-1-yl)-3,3a,4,6a-tetrahydro-2H-cyclopenta[ $d]$ oxazol-2-one (5b)

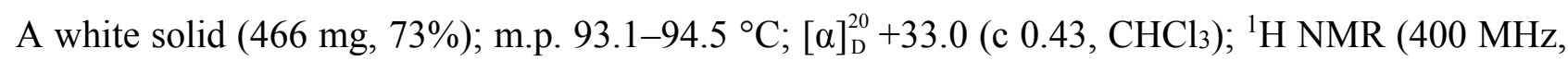
$\left.\mathrm{CDCl}_{3}\right) \delta 6.12(\mathrm{~d}, J=15.9 \mathrm{~Hz}, 1 \mathrm{H}), 5.94(\mathrm{td}, J=7.3 \mathrm{~Hz}, 15.1 \mathrm{~Hz}, 1 \mathrm{H}), 5.68(\mathrm{~s}, 1 \mathrm{H}), 5.59(\mathrm{~d}$, $J=7.7 \mathrm{~Hz}, 1 \mathrm{H}), 5.51$ (brs, 1H), 4.44 (t, $J=7.1 \mathrm{~Hz}, 1 \mathrm{H}), 2.72$ (dd, $J=6.0 \mathrm{~Hz}, 18.3 \mathrm{~Hz}, 1 \mathrm{H}), 2.47$ (d, $J=18.2 \mathrm{~Hz}, 1 \mathrm{H}), 2.13-2.04(\mathrm{~m}, 2 \mathrm{H}), 1.40-1.37(\mathrm{~m}, 2 \mathrm{H}), 1.24(\mathrm{~s}, 18 \mathrm{H}), 0.86(\mathrm{t}, J=6.7 \mathrm{~Hz}, 3 \mathrm{H})$; ${ }^{13} \mathrm{C} \mathrm{NMR}\left(100 \mathrm{MHz}, \mathrm{CDCl}_{3}\right) \delta 158.8,139.8,135.1,128.7,123.0,84.9,53.7,40.2,33.1,31.9,29.7$ (2C), 29.64, 29.58, 29.48, 29.35, 29.3, 29.0, 22.7, 14.1; IR ( $\left.\mathrm{CHCl}_{3}\right) v_{\max } 3244,2917,2852,1735,1471,1386$, 1248, 1016, $716\left(\mathrm{~cm}^{-1}\right)$; HRMS (FAB) calcd. for $\mathrm{C}_{20} \mathrm{H}_{34} \mathrm{NO}_{2}\left([\mathrm{M}+\mathrm{H}]^{+}\right), 320.2590$; found, 320.2584.

3.1.9. (3aS,6aR)-6-((E)-Hexadec-1-en-1-yl)-3,3a,4,6a-tetrahydro-2H-cyclopenta[d]oxazol-2-one (5c)

A white solid (466 mg, 67\%); m.p. 90.1-91.6 ${ }^{\circ} \mathrm{C} ;[\alpha]_{\mathrm{D}}^{20}+38.4\left(\mathrm{c} 0.95, \mathrm{CHCl}_{3}\right) ;{ }^{1} \mathrm{H} \mathrm{NMR}(600 \mathrm{MHz}$, $\left.\mathrm{CDCl}_{3}\right) \delta 6.12(\mathrm{~d}, J=16.0 \mathrm{~Hz}, 1 \mathrm{H}), 5.94(\mathrm{td}, J=7.4 \mathrm{~Hz}, 15.3 \mathrm{~Hz}, 1 \mathrm{H}), 5.86(\mathrm{brs}, 1 \mathrm{H}), 5.68(\mathrm{~s}, 1 \mathrm{H})$, 5.59 (d, $J=8.2 \mathrm{~Hz}, 1 \mathrm{H}), 4.44(\mathrm{t}, J=7.1 \mathrm{~Hz}, 1 \mathrm{H}), 2.70$ (dd, $J=6.4 \mathrm{~Hz}, 17.9 \mathrm{~Hz}, 1 \mathrm{H}), 2.47$ (d, $J=18.3 \mathrm{~Hz}, 1 \mathrm{H}), 2.15-2.02(\mathrm{~m}, 2 \mathrm{H}), 1.39-1.34(\mathrm{~m}, 2 \mathrm{H}), 1.24(\mathrm{~s}, 22 \mathrm{H}), 0.86(\mathrm{t}, J=7.1 \mathrm{~Hz}, 3 \mathrm{H})$;

${ }^{13} \mathrm{C} \mathrm{NMR}\left(150 \mathrm{MHz}, \mathrm{CDCl}_{3}\right) \delta 159.0,139.8,135.0,128.9,123.0,84.9,53.8,40.2,33.1,31.9,29.67$ (3C), 29.64 (2C), 29.58, 29.48, 29.35, 29.26, 29.0, 22.7, 14.1; IR $\left(\mathrm{CHCl}_{3}\right) v_{\max } 3284,2922,2853$, 1737, 1716, 1385, 1237, $1015\left(\mathrm{~cm}^{-1}\right)$; HRMS (FAB) calcd. for $\mathrm{C}_{22} \mathrm{H}_{38} \mathrm{NO}_{2}\left([\mathrm{M}+\mathrm{H}]^{+}\right), 348.2903$; found, 348.2905 .

\subsubsection{General Procedure for the Preparation of 11}

To a solution of diene $5(0.72 \mathrm{mmol})$ in $\mathrm{MeOH}(30 \mathrm{~mL})$ was added $\mathrm{Pd} / \mathrm{C}(40 \mathrm{mg}, 10 \mathrm{wt} . \% \mathrm{Pd})$ at room temperature. The reaction mixture was shaken in a Parr hydrogenator for $10 \mathrm{~h}$ at an initial pressure of 35 psi. The catalyst was filtered off with Celite ${ }^{\circledR}$ (OCI Company Ltd., Seoul, Korea), and the solvent was removed under reduced pressure. The crude product was purified by column chromatography on silica gel (hexane/EtOAc, 1:3) to give the desired product, 11.

\subsubsection{1. (3a $S, 6 R, 6 \mathrm{a} R)$-6-Dodecylhexahydro-2H-cyclopenta[ $d]$ oxazol-2-one (11a)}

A white solid (117 mg, 55\%). m.p. 67.8-69.1 ${ }^{\circ} \mathrm{C} ;[\alpha]_{\mathrm{D}}^{20}+10.6\left(\mathrm{c} 0.50, \mathrm{CHCl}_{3}\right) ;{ }^{1} \mathrm{H}$ NMR (400 MHz, $\left.\mathrm{CDCl}_{3}\right) \delta 5.71$ (brs, $\left.1 \mathrm{H}\right), 4.88(\mathrm{t}, J=6.1 \mathrm{~Hz}, 1 \mathrm{H}), 4.22(\mathrm{t}, J=6.5 \mathrm{~Hz}, 1 \mathrm{H}), 1.76-1.69(\mathrm{~m}, 3 \mathrm{H})$, $1.63-1.55(\mathrm{~m}, 2 \mathrm{H}), 1.53-1.39(\mathrm{~m}, 2 \mathrm{H}), 1.23(\mathrm{~s}, 20 \mathrm{H}), 0.86(\mathrm{t}, J=6.7 \mathrm{~Hz}, 3 \mathrm{H}) ;{ }^{13} \mathrm{C} \mathrm{NMR}(100 \mathrm{MHz}$, $\left.\mathrm{CDCl}_{3}\right) \delta 160.1,83.2,56.6,46.0,33.8,31.9,29.8,29.62$ (4C), 29.56, 29.3, 28.4, 28.2, 28.0, 22.7, 14.1; IR $\left(\mathrm{CHCl}_{3}\right) v_{\max } 3306,2923,2854,1736,1713,1423,1242\left(\mathrm{~cm}^{-1}\right)$; HRMS (FAB) calcd. for $\mathrm{C}_{18} \mathrm{H}_{34} \mathrm{NO}_{2}\left([\mathrm{M}+\mathrm{H}]^{+}\right), 296.2590$; found, 296.2588 .

\subsubsection{2. (3aS,6R,6aR)-6-Tetradecylhexahydro-2H-cyclopenta[ $[d]$ oxazol-2-one (11b)}

A white solid (127 mg, 57\%). m.p. 88.7-89.6 ${ }^{\circ} \mathrm{C} ;[\alpha]_{\mathrm{D}}^{20}+8.56\left(\mathrm{c} 0.52, \mathrm{CHCl}_{3}\right) ;{ }^{1} \mathrm{H}$ NMR (400 MHz, $\left.\mathrm{CDCl}_{3}\right) \delta 5.59$ (brs, $\left.1 \mathrm{H}\right), 4.88(\mathrm{t}, J=6.1 \mathrm{~Hz}, 1 \mathrm{H}), 4.23(\mathrm{t}, J=6.5 \mathrm{~Hz}, 1 \mathrm{H}), 1.77-1.70(\mathrm{~m}, 3 \mathrm{H})$, 
1.60-1.54 (m, 2H), 1.48-1.42 (m, 2H), $1.23(\mathrm{~s}, 24 \mathrm{H}), 0.86(\mathrm{t}, J=6.8 \mathrm{~Hz}, 3 \mathrm{H}) ;{ }^{13} \mathrm{C} \mathrm{NMR}(100 \mathrm{MHz}$, $\left.\mathrm{CDCl}_{3}\right) \delta 160.0,83.2,56.6,46.1,33.9,31.9,29.8,29.67$ (3C), 29.64 (3C), 29.57, 29.3, 28.4, 28.3, 28.0, 22.7, 14.1; IR $\left(\mathrm{CHCl}_{3}\right) v_{\max } 3255,2919,2852,1723,1469,1248\left(\mathrm{~cm}^{-1}\right)$; HRMS (FAB) calcd. for $\mathrm{C}_{20} \mathrm{H}_{38} \mathrm{NO}_{2}\left([\mathrm{M}+\mathrm{H}]^{+}\right), 324.2903$; found, 324.2903 .

\subsubsection{3. (3aS,6R,6aR)-6-Hexadecylhexahydro-2H-cyclopenta[ $d]$ oxazol-2-one (11c)}

A white solid (122 mg, 50\%). m.p. 91.9-92.1 ${ }^{\circ} \mathrm{C} ;[\alpha]_{\mathrm{D}}^{20}+7.91\left(\mathrm{c} 0.46, \mathrm{CHCl}_{3}\right) ;{ }^{1} \mathrm{H}$ NMR (400 MHz, $\left.\mathrm{CDCl}_{3}\right) \delta 5.51$ (brs, $\left.1 \mathrm{H}\right), 4.89(\mathrm{t}, J=6.1 \mathrm{~Hz}, 1 \mathrm{H}), 4.23(\mathrm{t}, J=6.5 \mathrm{~Hz}, 1 \mathrm{H}), 1.77-1.70(\mathrm{~m}, 3 \mathrm{H}), 1.61-1.55$ $(\mathrm{m}, 2 \mathrm{H}), 1.48-1.40(\mathrm{~m}, 2 \mathrm{H}), 1.23(\mathrm{~s}, 28 \mathrm{H}), 0.86(\mathrm{t}, J=6.8 \mathrm{~Hz}, 3 \mathrm{H}) ;{ }^{13} \mathrm{C} \mathrm{NMR}\left(100 \mathrm{MHz}, \mathrm{CDCl}_{3}\right)$ $\delta 159.9,83.2,56.6,46.1,33.9,31.9,29.8,29.68$ (5C), 29.65 (3C), 29.58, 29.3, 28.4, 28.3, 28.0, 22.7, 14.1; IR $\left(\mathrm{CHCl}_{3}\right) v_{\max } 3262,2918,2851,1725,1469,1245\left(\mathrm{~cm}^{-1}\right)$; HRMS (FAB) calcd. for $\mathrm{C}_{22} \mathrm{H}_{42} \mathrm{NO}_{2}$ $\left([\mathrm{M}+\mathrm{H}]^{+}\right), 352.3216$; found, 352.3216 .

\subsubsection{General Procedure for the Preparation of 4}

To a solution of $11(0.071 \mathrm{mmol})$ in $\mathrm{EtOH}(1 \mathrm{~mL})$ was added $1 \mathrm{M}$ aq $\mathrm{KOH}(1 \mathrm{~mL})$ at room temperature. The reaction mixture was stirred for $20 \mathrm{~h}$ at $85{ }^{\circ} \mathrm{C}$. After the reaction mixture was cooled to room temperature, the solvent was removed under reduced pressure. The crude product was purified by column chromatography on silica gel $\left(\mathrm{CH}_{2} \mathrm{Cl}_{2} / \mathrm{MeOH} / \mathrm{NH}_{4} \mathrm{OH}, 100: 10: 1\right)$ to give the desired product, 4 .

\subsubsection{5. (1R,2S,5R)-2-Amino-5-dodecylcyclopentan-1-ol (4a)}

A white solid (17 mg, 88\%). m.p. 93.2-96.2 ${ }^{\circ} \mathrm{C}$; $[\alpha]_{\mathrm{D}}^{20}+3.96$ (c 0.36, EtOH); ${ }^{1} \mathrm{H}$ NMR (400 MHz, $\left.\mathrm{CDCl}_{3}\right) \delta 3.68(\mathrm{t}, J=3.5 \mathrm{~Hz}, 1 \mathrm{H}), 3.30(\mathrm{td}, J=3.8 \mathrm{~Hz}, 8.4 \mathrm{~Hz}, 1 \mathrm{H}), 2.10(\mathrm{brs}, 2 \mathrm{H}), 1.91-1.86(\mathrm{~m}, 1 \mathrm{H})$, 1.74-1.66 (m, 2H), 1.56-1.34 (m, 1H), $1.23(\mathrm{~s}, 23 \mathrm{H}), 0.86(\mathrm{t}, J=6.8 \mathrm{~Hz}, 3 \mathrm{H}) ;{ }^{13} \mathrm{C}$ NMR $(100 \mathrm{MHz}$, $\left.\mathrm{CDCl}_{3}\right) \delta 74.4,55.4,44.2,31.9,30.9,29.95,29.93,29.68$ (3C), 29.64, 29.3, 28.5, 28.0, 22.7, 14.1; IR $\left(\mathrm{CHCl}_{3}\right) v_{\max }$ 3054, 2922, 2852, 2727, 1469, $992\left(\mathrm{~cm}^{-1}\right)$; HRMS (FAB) calcd. for $\mathrm{C}_{17} \mathrm{H}_{36} \mathrm{NO}$ $\left([\mathrm{M}+\mathrm{H}]^{+}\right), 270.2797$; found, 270.2794 .

\subsubsection{6. (1R,2S,5R)-2-Amino-5-tetradecylcyclopentan-1-ol (4b)}

A white solid (19 mg, 90\%). m.p. $100.1-101.0^{\circ} \mathrm{C} ;[\alpha]_{\mathrm{D}}^{20}+3.29$ (c 0.25, EtOH); ${ }^{1} \mathrm{H}$ NMR (400 MHz, $\left.\mathrm{CDCl}_{3}\right) \delta 3.70(\mathrm{t}, J=3.4 \mathrm{~Hz}, 1 \mathrm{H}), 3.30(\mathrm{td}, J=3.9 \mathrm{~Hz}, 8.5 \mathrm{~Hz}, 1 \mathrm{H}), 2.44$ (brs, 3H), 1.91-1.85 (m, 1H), $1.75-1.65(\mathrm{~m}, 2 \mathrm{H}), 1.54-1.34(\mathrm{~m}, 1 \mathrm{H}), 1.23(\mathrm{~s}, 27 \mathrm{H}), 0.86(\mathrm{t}, J=6.8 \mathrm{~Hz}, 3 \mathrm{H}) ;{ }^{13} \mathrm{C}$ NMR $(100 \mathrm{MHz}$, $\left.\mathrm{CDCl}_{3}\right) \delta 74.4,55.4,44.1,31.9,30.8,30.0,29.9,29.69$ (5C), 29.65 (2C), 29.3, 28.5, 28.0, 22.7, 14.1; IR $\left(\mathrm{CHCl}_{3}\right) v_{\max }$ 3049, 2921, 2852, 2729, 1469, $994\left(\mathrm{~cm}^{-1}\right)$; HRMS (FAB) calcd. for $\mathrm{C}_{19} \mathrm{H}_{40} \mathrm{NO}$ $\left([\mathrm{M}+\mathrm{H}]^{+}\right), 298.3110$; found, 298.3117 .

\subsubsection{7. (1R,2S,5R)-2-Amino-5-hexadecylcyclopentan-1-ol (4c)}

A white solid (21 mg, 91\%). m.p. $102.1-103.0{ }^{\circ} \mathrm{C} ;[\alpha]_{\mathrm{D}}^{20}+1.89\left(\mathrm{c} 0.51, \mathrm{CHCl}_{3}\right) ;{ }^{1} \mathrm{H} \mathrm{NMR}(400 \mathrm{MHz}$, $\left.\mathrm{CDCl}_{3}\right) \delta 3.70(\mathrm{t}, J=3.5 \mathrm{~Hz}, 1 \mathrm{H}), 3.30(\mathrm{td}, J=3.8 \mathrm{~Hz}, 8.2 \mathrm{~Hz}, 1 \mathrm{H}), 2.41$ (brs, 3H), 1.89-1.85 (m, 1H), $1.75-1.65(\mathrm{~m}, 2 \mathrm{H}), 1.54-1.34(\mathrm{~m}, 1 \mathrm{H}), 1.23(\mathrm{~s}, 31 \mathrm{H}), 0.86(\mathrm{t}, J=6.8 \mathrm{~Hz}, 3 \mathrm{H}) ;{ }^{13} \mathrm{C}$ NMR $(100 \mathrm{MHz}$, 
$\left.\mathrm{CDCl}_{3}\right) \delta$ 74.5, 55.5, 44.2, 31.9, 30.9, 30.0, 29.9, 29.69 (7C), 29.65 (2C), 29.4, 28.5, 28.0, 22.7, 14.1; IR $\left(\mathrm{CHCl}_{3}\right) v_{\max } 3057,2921,2852,2728,1468,995\left(\mathrm{~cm}^{-1}\right)$; HRMS (FAB) calcd. for $\mathrm{C}_{21} \mathrm{H}_{44} \mathrm{NO}$ $\left([\mathrm{M}+\mathrm{H}]^{+}\right), 326.3423$; found, 326.3433 .

\subsection{Biological Evaluation}

\subsubsection{Sulforhodamine B (SRB) Assay}

Cells $\left(5 \times 10^{4}\right.$ cells $\left./ \mathrm{mL}\right)$ were treated with various concentrations of test compounds in 96-well culture plates for $72 \mathrm{~h}$. After incubation, cells were fixed with 10\% trichloroacetic acid (TCA), dried and stained with $0.4 \% \mathrm{SRB}$ in $1 \%$ acetic acid. The unbound dye was washed out, and the stained cells were dried and resuspended in $10 \mathrm{mM}$ Tris (pH 10.0). The absorbance at $515 \mathrm{~nm}$ was measured, and cell proliferation was determined as follows: cell proliferation $(\%)=\left(\right.$ average absorbance compound $_{-}$ average absorbance day zero $) /\left(\right.$ average absorbance control $_{-}$average absorbance day zero $) \times 100$. IC $_{50}$ values were calculated by nonlinear regression analysis using TableCurve 2D v5.01 (Systat Software Inc., Richmond, CA, USA).

\subsubsection{Sphingosine Kinase Inhibition Assay}

In 384-well polystyrene plates, Sphk1 and Sphk2 (BPS Bioscience, San Diego, CA, USA) were incubated in kinase buffer (5 mM MOPS, pH 7.2, $2.5 \mathrm{mM} \beta$-glycerol-phosphate, $5 \mathrm{mM} \mathrm{MgCl}_{2}$, $1 \mathrm{mM}$ EGTA, $0.4 \mathrm{mM}$ EDTA, $0.5 \mathrm{mM}$ DTT) containing $1 \mu \mathrm{M}$ sphingosine, ATP (50 $\mu \mathrm{M}$ for SphK1; $150 \mu \mathrm{M}$ for SphK2) and test compounds with a final concentration of $1 \%$ DMSO for $1 \mathrm{~h}$ at room temperature. The amount of ATP transferred was measured with the ADP-Glo ${ }^{\text {TM }}$ kinase assay kit (Catalog \#V9101, Promega, Madison, WI, USA) according to the manufacturer's instructions. IC50 values were calculated using GraphPad Prism 5 software (GraphPad Software Inc., La Jolla, CA, USA).

\subsection{Molecular Modeling}

To understand the binding mode of the pachastrissamine and its analogue, we performed a flexible docking study using the Schrödinger Glide program with standard precision settings (Schrödinger, LLC, New York, NY, USA, http://www.schrodinger.com). The X-ray crystal structure of the human SphK1 (PDB Code 3VZB) was obtained from the Protein Data Bank (PDB, [36]). The pachastrissamine and 4b were minimized using a Merck Molecular Force Field (MMFF) with a dielectric constant of 80.0 using the MacroModel program suite. The initial structure of pachastrissamine and $\mathbf{4 b}$ were built based on the sphingosine structure co-crystallized with SphK1. The best docking result was visualized using Discovery Studio 4.1 (Accelrys Software, Inc., San Diego, CA, USA). The hydrogen bonding interactions were displayed as green dashed lines between the ligand and the SphK1.

\section{Conclusions}

We have reported the synthesis and biological evaluation of the carbocyclic analogues of pachastrissamine with varying chain lengths. The designed carbocyclic analogues were efficiently 
synthesized in good overall yield by the tandem ene/yne-ene metathesis as a key step. Among them, the analogue $\mathbf{4 b}$ exhibits comparable cytotoxic activity and more potent inhibitory activity against sphingosine kinases, compared to the parent natural product. In our docking model, 4b showed an additional interaction caused by the adjacent hydrophobic amino acid residue, which could explain its increased inhibitory activity. These results imply that bioisosteric replacement of the ethereal oxygen in the cyclic core to the methylene carbon is possible. Our findings are valuable in the development of sphingosine kinase inhibitors, which may lead to the development of promising new anti-cancer therapeutic agents. Further studies toward the biochemical and pharmacological properties of the carbocyclic analogue, based on these findings, are worthy of pursuing in the future.

\section{Acknowledgments}

This work was supported by the Mid-Career Researcher Program (No. 2013R1A2A1A01015998) of the National Research Foundation of Korea (NRF) grant funded by the Korean government (MSIP). This work was also supported by an NRF (National Research Foundation of Korea) grant funded by the Korean Government (NRF-2012-Fostering Core Leaders of the Future Basic Science Program).

\section{Author Contributions}

Yongseok Kwon, Hoon Bae and Geun-Hee Han performed the synthetic experiments. Jayoung Song and Sang Kook Lee performed the biological experiments. Joo-Youn Lee performed the molecular modeling. Yongseok Kwon, Woo-Jung Kim and Sanghee Kim wrote the manuscript. Sanghee Kim designed and developed the study.

\section{Conflicts of Interest}

The authors declare no conflict of interest.

\section{References}

1. Kuroda, I.; Musman, M.; Ohtani, I.I.; Ichiba, T.; Tanaka, J.; Gravalos, D.G.; Higa, T. Pachastrissamine, a cytotoxic anhydrophytosphingosine from a marine sponge, Pachastrissa sp. J. Nat. Prod. 2002, 65, 1505-1506.

2. Ledroit, V.; Debitus, C.; Lavaud, C.; Massiot, G. Jaspines A and B: Two new cytotoxic sphingosine derivatives from the marine sponge Jaspis sp. Tetrahedron Lett. 2003, 44, 225-228.

3. Liu, J.; Du, Y.; Dong, X.; Meng, S.; Xiao, J.; Cheng, L. Stereoselective synthesis of jaspine B from D-xylose. Carbohydr. Res. 2006, 341, 2653-2657.

4. Salma, Y.; Lafont, E.; Therville, N.; Carpentier, S.; Bonnafé, M.-J.; Levade, T.; Génisson, Y.; Andrieu-Abadie, N. The natural marine anhydrophytosphingosine, jaspine B, induces apoptosis in melanoma cells by interfering with ceramide metabolism. Biochem. Pharmacol. 2009, 78, 477-485.

5. Yoshimitsu, Y.; Oishi, S.; Miyagaki, J.; Inuki, S.; Ohno, H.; Fujii, N. Pachastrissamine (jaspine B) and its stereoisomers inhibit sphingosine kinases and atypical protein kinase C. Biorg. Med. Chem. 2011, 19, 5402-5408. 
6. Inuki, S.; Yoshimitsu, Y.; Oishi, S.; Fujii, N.; Ohno, H. Ring-construction/stereoselective functionalization cascade: Total synthesis of pachastrissamine (jaspine B) through palladium-catalyzed bis-cyclization of propargyl chlorides and carbonates. J. Org. Chem. 2010, 75, 3831-3842.

7. Passiniemi, M.; Koskinen, A.M.P. Asymmetric synthesis of pachastrissamine (jaspine B) and its diastereomers via $\eta^{3}$-allylpalladium intermediates. Org. Biomol. Chem. 2011, 9, 1774-1783.

8. Srinivas Rao, G.; Venkateswara Rao, B. A common strategy for the stereoselective

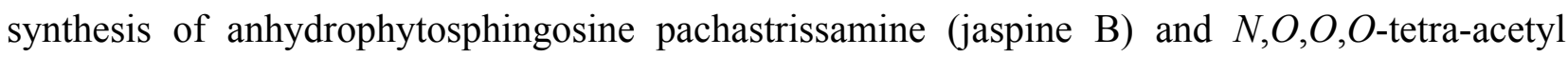
D-lyxo-phytosphingosine. Tetrahedron Lett. 2011, 52, 6076-6079.

9. Llaveria, J.; Díaz, Y.; Matheu, M.I.; Castillón, S. Enantioselective synthesis of jaspine B (pachastrissamine) and its C-2 and/or C-3 epimers. Eur. J. Org. Chem. 2011, 1514-1519.

10. Zhao, M.-L.; Zhang, E.; Gao, J.; Zhang, Z.; Zhao, Y.-T.; Qu, W.; Liu, H.-M. An efficient and convenient formal synthesis of jaspine B from D-xylose. Carbohydr. Res. 2012, 351, 126-129.

11. Schmiedel, V.M.; Stefani, S.; Reissig, H.-U. Stereodivergent synthesis of jaspine B and its isomers using a carbohydrate-derived alkoxyallene as C3-building block. Beilstein J. Org. Chem. 2013, 9, 2564-2569.

12. Ghosal, P.; Ajay, S.; Meena, S.; Sinha, S.; Shaw, A.K. Stereoselective total synthesis of jaspine B (pachastrissamine) utilizing iodocyclization and an investigation of its cytotoxic activity. Tetrahedron Asymmetry 2013, 24, 903-908.

13. Jana, A.K.; Panda, G. Stereoselective synthesis of jaspine B and its C2 epimer from garner aldehyde. RSC Adv. 2013, 3, 16795-16801.

14. Dhand, V.; Chang, S.; Britton, R. Total synthesis of the cytotoxic anhydrophytosphingosine pachastrissamine (jaspine B). J. Org. Chem. 2013, 78, 8208-8213.

15. Lin, C.-W.; Liu, S.-W.; Hou, D.-R. Formation of tetrahydrofurans via a 5-endo-tet cyclization of aziridines-Synthesis of (-)-pachastrissamine. Org. Biomol. Chem. 2013, 11, 5292-5299.

16. Martinková, M.; Mezeiová, E.; Gonda, J.; Jacková, D.; Pomikalová, K. Total synthesis of (-)-jaspine B and its 4-epi-analogue from D-xylose. Tetrahedron Asymmetry 2014, 25, 750-766.

17. Génisson, Y.; Lamandé, L.; Salma, Y.; Andrieu-Abadie, N.; André, C.; Baltas, M. Enantioselective access to a versatile 4-oxazolidinonecarbaldehyde and application to the synthesis of a cytotoxic jaspine B truncated analogue. Tetrahedron Asymmetry 2007, 18, 857-864.

18. Salma, Y.; Ballereau, S.; Maaliki, C.; Ladeira, S.; Andrieu-Abadie, N.; Génisson, Y. Flexible and enantioselective access to jaspine $\mathrm{B}$ and biologically active chain-modified analogues thereof. Org. Biomol. Chem. 2010, 8, 3227-3243.

19. Canals, D.; Mormeneo, D.; Fabriàs, G.; Llebaria, A.; Casas, J.; Delgado, A. Synthesis and biological properties of pachastrissamine (jaspine B) and diastereoisomeric jaspines. Biorg. Med. Chem. 2009, 17, 235-241.

20. Xu, J.-M.; Zhang, E.; Shi, X.-J.; Wang, Y.-C.; Yu, B.; Jiao, W.-W.; Guo, Y.-Z.; Liu, H.-M. Synthesis and preliminary biological evaluation of 1,2,3-triazole-jaspine B hybrids as potential cytotoxic agents. Eur. J. Med. Chem. 2014, 80, 593-604.

21. Rives, A.; Ladeira, S.; Levade, T.; Andrieu-Abadie, N.; Génisson, Y. Synthesis of cytotoxic aza analogues of jaspine B. J. Org. Chem. 2010, 75, 7920-7923. 
22. Jeon, H.; Bae, H.; Baek, D.J.; Kwak, Y.-S.; Kim, D.; Kim, S. Syntheses of sulfur and selenium analogues of pachastrissamine via double displacements of cyclic sulfate. Org. Biomol. Chem. 2011, 9, 7237-7242.

23. Patani, G.A.; LaVoie, E.J. Bioisosterism: A rational approach in drug design. Chem. Rev. 1996, 96, 3147-3176.

24. Arjona, O.; Gómez, A.M.; López, J.C.; Plumet, J. Synthesis and conformational and biological aspects of carbasugars. Chem. Rev. 2007, 107, 1919-2036.

25. Ferrero, M.; Gotor, V. Biocatalytic selective modifications of conventional nucleosides, carbocyclic nucleosides, and C-nucleosides. Chem. Rev. 2000, 100, 4319-4348.

26. Villar, H.; Frings, M.; Bolm, C. Ring closing enyne metathesis: A powerful tool for the synthesis of heterocycles. Chem. Soc. Rev. 2007, 36, 55-66.

27. Grubbs, R.H. Olefin metathesis. Tetrahedron 2004, 60, 7117-7140.

28. Royer, F.; Vilain, C.; Elkaïm, L.; Grimaud, L. Selective domino ring-closing metathesis-cross-metathesis reactions between enynes and electron-deficient alkenes. Org. Lett. 2003, 5, 2007-2009.

29. Lee, H.-Y.; Kim, H.Y.; Tae, H.; Kim, B.G.; Lee, J. One-pot three-component tandem metathesis/Diels-alder reaction. Org. Lett. 2003, 5, 3439-3442.

30. Borzilleri, R.M.; Zheng, X.; Schmidt, R.J.; Johnson, J.A.; Kim, S.-H.; DiMarco, J.D.; Fairchild, C.R.; Gougoutas, J.Z.; Lee, F.Y.F.; Long, B.H.; et al. A novel application of a $\operatorname{Pd}(0)$-catalyzed nucleophilic substitution reaction to the regio- and stereo-selective synthesis of lactam analogues of the epothilone natural products. J. Am. Chem. Soc. 2000, 122, 8890-8897.

31. Lee, S.K.; Nam, K.-A.; Heo, Y.-H. Cytotoxic activity and G2/M cell cycle arrest mediated by antofine, a phenanthroindolizidine alkaloid isolated from cynanchum paniculatum. Planta Med. 2003, 69, 21-25.

32. Pyne, N.J.; Pyne, S. Sphingosine 1-phosphate and cancer. Nat. Rev. Cancer 2010, 10, 489-503.

33. Yatomi, Y.; Ruan, F.; Megidish, T.; Toyokuni, T.; Hakomori, S.-I.; Igarashi, Y. $N, N$-dimethylsphingosine inhibition of sphingosine kinase and sphingosine 1-phosphate activity in human platelets. Biochemistry 1996, 35, 626-633.

34. Wang, Z.; Min, X.; Xiao, S.-H.; Johnstone, S.; Romanow, W.; Meininger, D.; Xu, H.; Liu, J.; Dai, J.; An, S.; et al. Molecular basis of sphingosine kinase 1 substrate recognition and catalysis. Structure 2013, 21, 798-809.

35. Bissantz, C.; Kuhn, B.; Stahl, M. A medicinal chemist's guide to molecular interactions. J. Med. Chem. 2010, 53, 5061-5084.

36. Protein Data Bank. Available online: http://www.rcsb.org (accessed on 27 November 2014).

(C) 2015 by the authors; licensee MDPI, Basel, Switzerland. This article is an open access article distributed under the terms and conditions of the Creative Commons Attribution license (http://creativecommons.org/licenses/by/4.0/). 